\title{
Electrical Impedance Spectroscopy combined with Temperature Cycled Operation for Semiconductor Gas Sensors
}

\author{
Andreas Schütze, Peter Reimann, Simon Darsch, Marco Schüler, Tobias Gillen \\ Laboratory for Measurement Technology, Saarland University, \\ Campus A5 1, 66123 Saarbrücken, Germany4
}

\begin{abstract}
Applications like fuel cells, batteries or food analysis are typical fields of interest for electrical impedance spectroscopy (EIS). Another field with promising results are EIS measurements in semiconductor (sc) gas sensor applications [1]. Due to their high sensitivity and low cost, sc gas sensors are ideally suited for different applications, especially in the field of safety and security. The major drawbacks of sc gas sensors, i.e. poor selectivity and sensor baseline drift, can be overcome by using temperature cycled operation (TCO) with DC measurement [2] or EIS, both in combination with intelligent signal processing. Currently, EIS for sc gas sensors is only possible in laboratory environments using commercial impedance analyzers, which are characterized by high cost, poor mobility and long acquisition time. However, for efficient development of application specific sc gas sensor systems and/or a self-monitoring strategy [3] extensive field tests are necessary. Therefore, a new hardware concept for EIS of Sc gas sensors based on FPGA-technology was developed. This concept also allows a combination of EIS with TCO for the first time. Additionally, state-of-the-art feature extraction methods are applied to sc gas sensor data and new features were tested [4].
\end{abstract}

\section{Motivation}

Due to their high sensitivity and low cost, semiconductor gas sensors are ideally suited for safety and security applications like fire and leakage detection $[5,6]$. The major drawbacks of this type of sensor are the poor selectivity, the sensor baseline drift and sensor poisoning. Especially for safety and security applications these drawbacks must be overcome to allow a proper function of the system. Temperature cycled operation (TCO) and electrical impedance spectroscopy (EIS) - both in combination with intelligent signal processing - are the main approaches for improving both selectivity and stability. In TCO mode the sensor temperature is changed periodically [2] and the DC resistance of the sensor is read-out continually during this cycle. For EIS the sensor temperature is usually kept constant and the sensor impedance is measured over a wide frequency range, e.g. during a frequency sweep [1,7,8]. To detect a sensor malfunction due to sensor poisoning a self-monitoring strategy can be realized by combining TCO und EIS (fig. 1) [3]. This strategy is based on redundant information from both modes which are evaluated separately and comparing both results. Additional information can be used for selectivity enhancement.

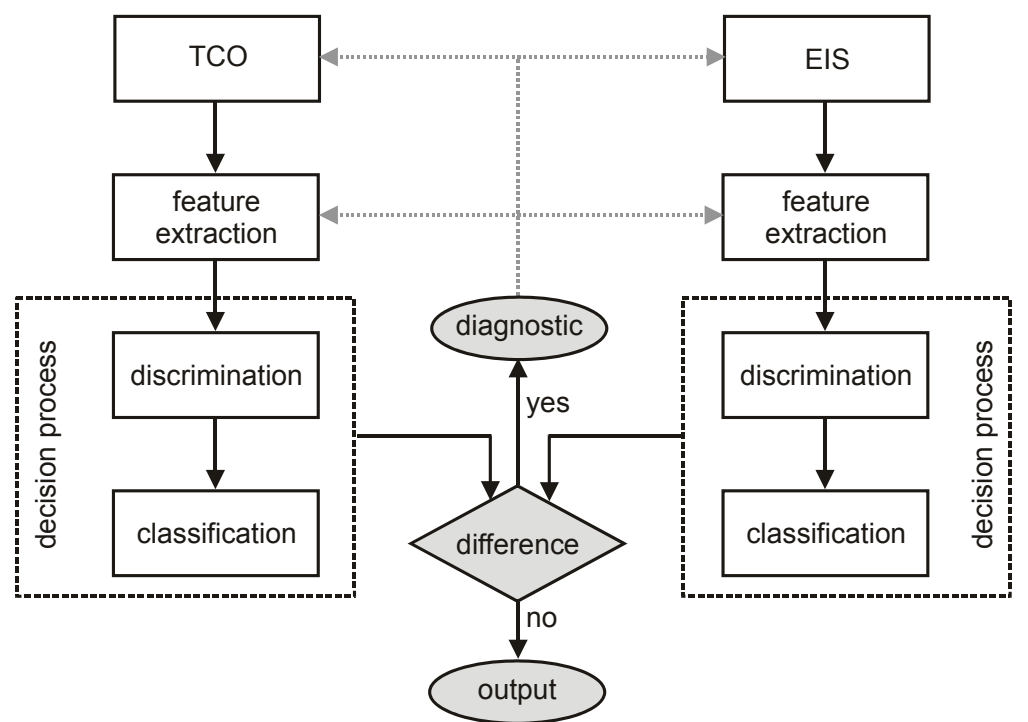

Fig. 1 Self-monitoring and self-diagnosis strategy for applications with semiconductor gas sensors: Redundant data from TCO and EIS are evaluated separately and the output is checked for differences [3]. 
For the TCO approach an adaptive and modular hardware was developed [9] and is currently being used in various lab und field tests [10]. For EIS measurements there is only a commercial lab-test system available with different drawbacks. The high cost, poor mobility and long acquisition times for sensor impedance spectra do not allow the use of this system in field tests and/or in combination with TCO. Especially for field tests a compact and low cost hardware has is required to make full use of this approach. In lab tests we calculate the sensor impedance by using the FFT after stimulating the sensor with a pseudo random binary sequence of maximum length (Maximum-Length-Sequence, MLS). The stimulation signal and the sensor response are both measured in the time domain. Based on the signal theory the sensor impedance is calculated by division of the stimulation and response spectra in the frequency domain. Whereas this approach was realized in the laboratory using a signal generator and an oscilloscope, in the newly developed platform signal generation and FFT are realized with an FPGA.

Closely connected with the hardware development and essential for complete measurement systems is an efficient feature extraction strategy for the gas sensor data.

\section{Experimental setup}

Currently, the impedance is measured using lab equipment based on a commercial EIS analyzer (Solartron SI 1210) [11]. This system allows an impedance sweep of 30 frequencies from $100 \mathrm{kHz}$ up to $32 \mathrm{MHz}$ in approximately one minute. Due to the long acquisition time, the sc sensor can only be operated in a static temperature mode, so that a combination of TCO and EIS cannot be realized with this setup. To overcome this limitation and based on intensive studies [12] we developed a measurement concept allowing the promising combination of both methods. Furthermore, this concept overcomes the drawbacks of poor mobility and high cost of the commercial EIS analyzer and, in addition, extends the frequency range up to $100 \mathrm{MHz}$. The overall concept is shown in fig. 2. The impedance of the device under test (DUT), i.e. the sc gas sensor, is determined by calculating the complex transfer function.

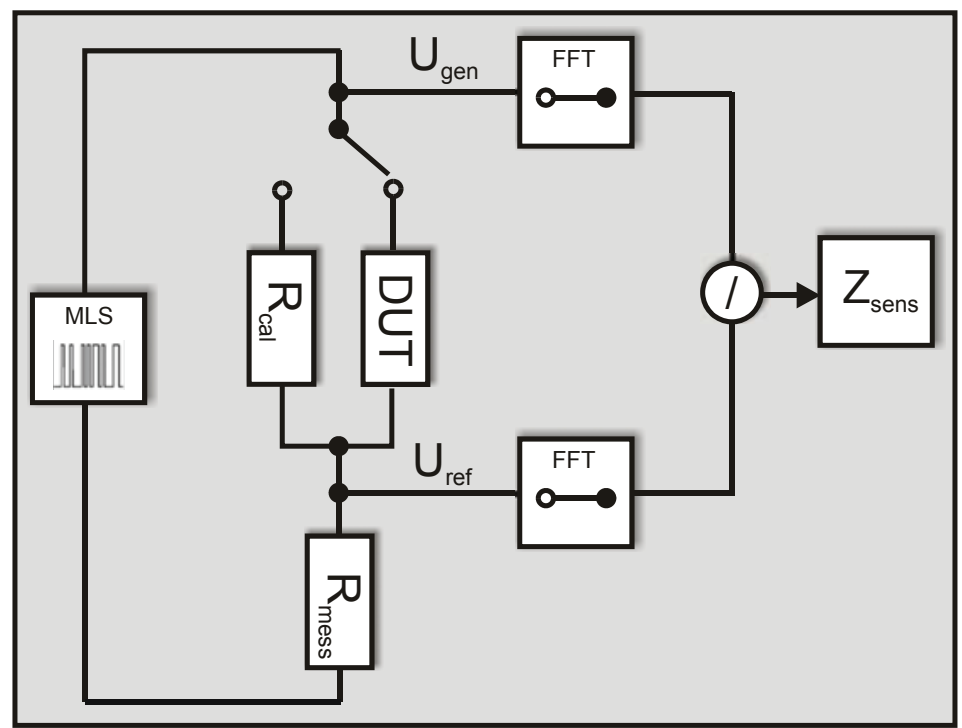

Fig. 2: Overall measurement concept for EIS based on FFT transformation.

In our first lab tests the sensor was stimulated using a commercial signal generator with a broad frequency spectrum. The stimulation signal $\left(U_{\text {gen }}\right)$ and the sensor response $\left(U_{\text {ref }}\right)$ were measured using a high speed oscilloscope. The impedance of the sensor was calculated by Fast Fourier Transformation (FFT) followed by dividing both spectra. After extensive tests with this setup and comparison of different stimulation signals (single pulse, white noise, Chirp, MLS) for EIS, we identified the Chirp and MLS signals as optimal for sensor stimulation [12]. A Chirp is a sinusoidal signal with continuously increasing frequency; the MLS (Maximum-Length-Sequence) is a type of pseudorandom binary sequence [13]. For efficient hardware implementation we decided to use the MLS signal, which requires only digital output instead of analog output for the Chirp signal. The major advantages of this approach are short measurement time and a relatively simple hardware setup.

First measurements with a commercial sc gas sensor (UST 1330 [14]) have shown promising results (fig. 3). The correlation between our FFT based concept and the results obtained with the commercial impedance analyzer is quite good. Different gases cause different amplitude and phase response and also a shift of the resonance frequency. To separate these gases relevant features have to be extracted. 


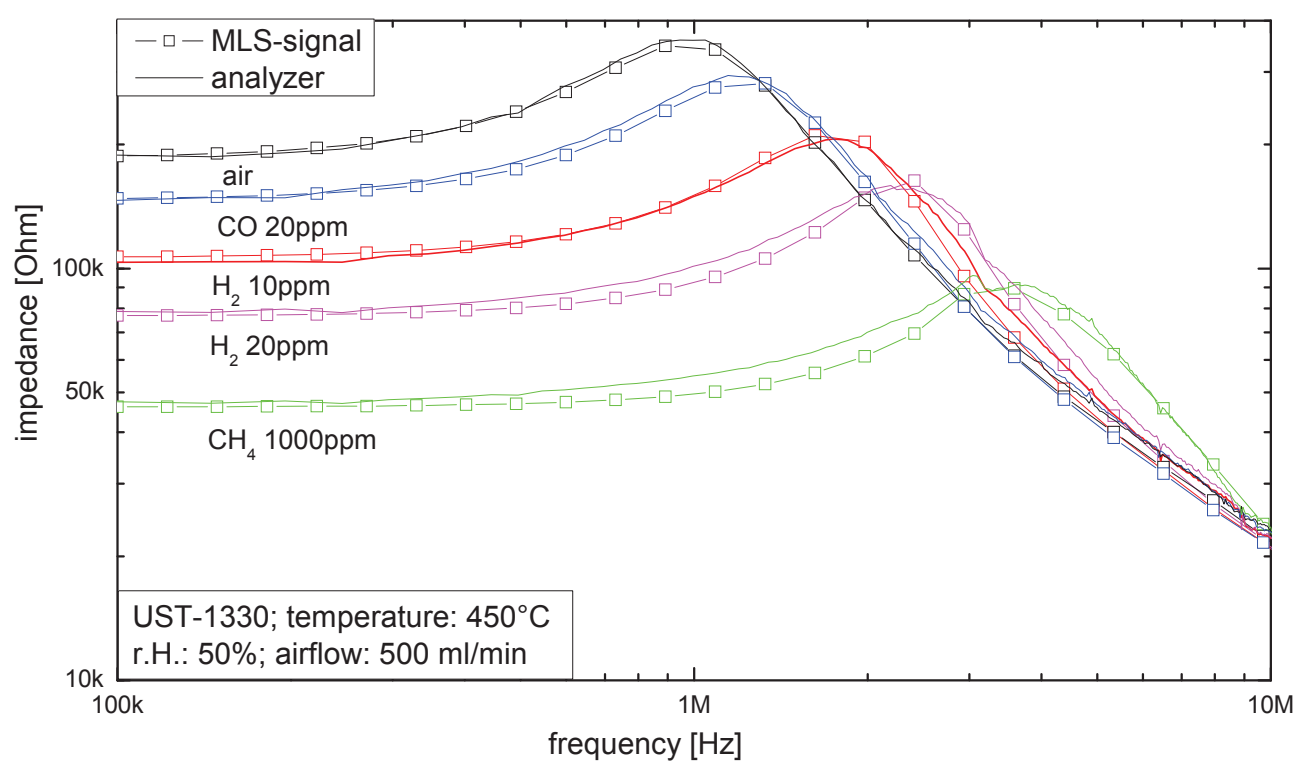

Fig. 3: Comparison of EIS spectra acquired using a commercial analyzer (solid line) and the FFT-based approach (squares).

\section{Results}

For evaluation of the FFT-based approach measurements in static temperature mode (STM) and TCO were done. In STM the sensor temperature is constant during measurement time and the impedance is measured. In TCO mode the temperature is changed while measuring the impedance. In both modes an MLS signal with a length of $21 \mu \mathrm{s}$ and a frequency range of $100 \mathrm{kHz}$ to $100 \mathrm{MHz}$ is used for determining the impedance. The different structure of the raw data resulted in different features which are the basis for the subsequent gas discrimination.

In STM the raw data are comparable with raw data from the commercial impedance analyzer, so that the proven feature extraction can be transferred. The features are separated in primary and secondary features. The primary features are calculated from the raw data in Bode and/or Nyquist plots. Prominent examples for features extracted from both plots are slopes, mean values or characteristic measurement points like minima or maxima as shown in fig. 6. For extraction of secondary features, the impedance curves are fitted for each sweep using equivalent circuit models [15]. Fig. 7 shows the equivalent circuit model composed of four parameters (two capacitances, one resistor and one inductivity) suitable for sc gas sensors. The extracted values of the circuit elements are the features used for the following data evaluation steps. Both primary and secondary features were evaluated for gas discrimination using Linear Discriminant Analysis (LDA) [16].
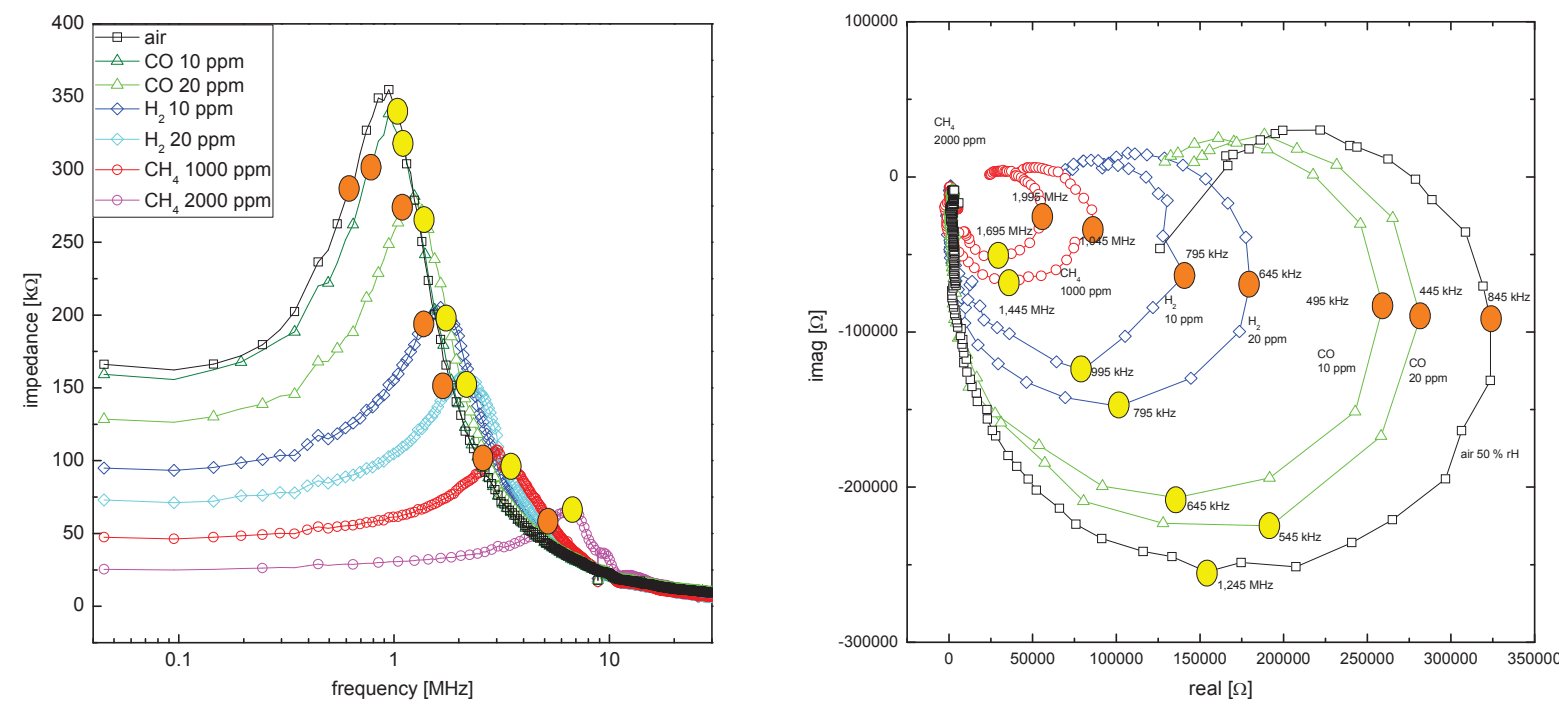

Fig. 6: Comparison of Bode and Nyquist plots with significant characteristic points and corresponding points. 


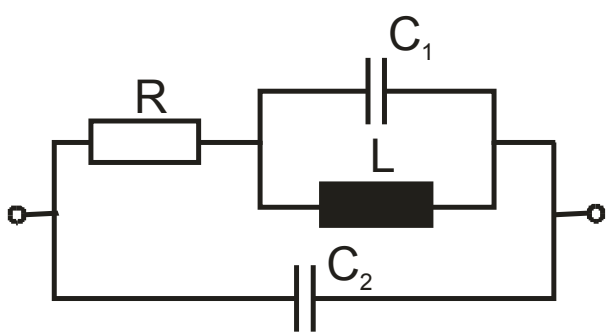

Fig. 7: Equivalent circuit model for fitting the measured impedance curves using the software LEVM

The feature extraction for EIS in TCO mode was not tested in previous studies. In our first tests the sC gas sensor is operated using a temperature cycle optimized for underground fire detection [5], which is also suitable for our test gases. In the cycle with an overall duration of $70.5 \mathrm{sec}$ the sensor is operated between $185^{\circ} \mathrm{C}$ and $387^{\circ} \mathrm{C}$. Based on the optimized EIS setup using FFT a complete impedance spectrum is measured every second. Thus, the raw data of a single temperature cycle contain 70 impedance spectra. The raw data patterns for air and $20 \mathrm{ppm} \mathrm{CO}$ are shown in fig. 8. The significant shapes can be described using the primary features described above.
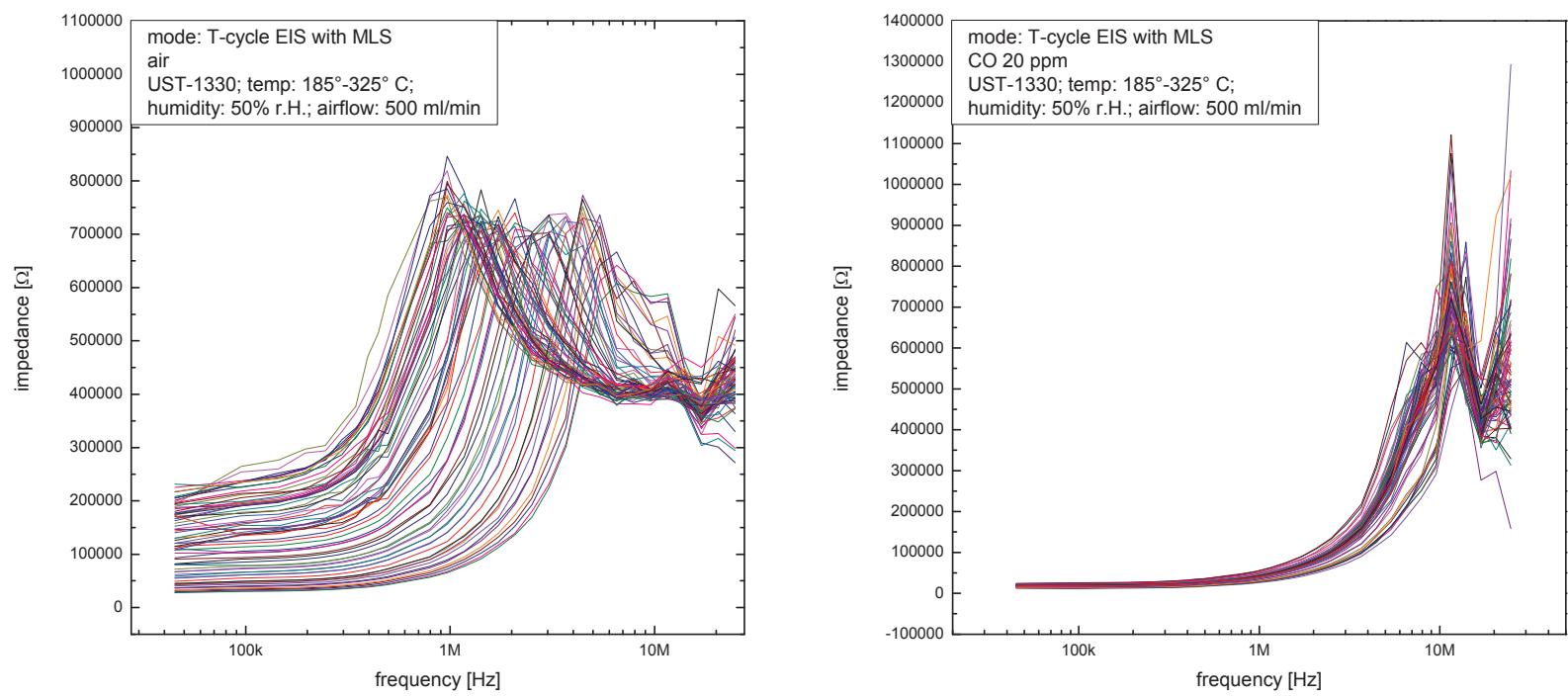

Fig. 8: Impedance spectra during a single temperature cycle for pure air (left) and air with 20 ppm CO (right). Both plots show 70 complete impedance spectra.

The best results for gas discrimination, however, were obtained with descriptive features, such as the area between the minimum and the maximum curve, the difference of the value at a defined frequency, the shift of the frequency maximum between certain curves or the averaging of the impedance over certain frequency ranges. These features are thus obtained not from a single but multiple spectra. Fig. 9 (right) shows the LDA based on the primary features from TCO. While the discrimination between air and the three test gases, especially $\mathrm{CH}_{4}$, is quite good, the discrimination between $\mathrm{H}_{2}$ and $\mathrm{CO}$ is rather poor and should be improved. To improve this discrimination the extraction of secondary features should be suitable. However, this is only possible with a higher data density requiring an improved hardware platform with higher sampling rate. Due to the comparatively low data density of the impedance spectra obtained in the current FFT mode set-up, the extraction of secondary features was not possible. 

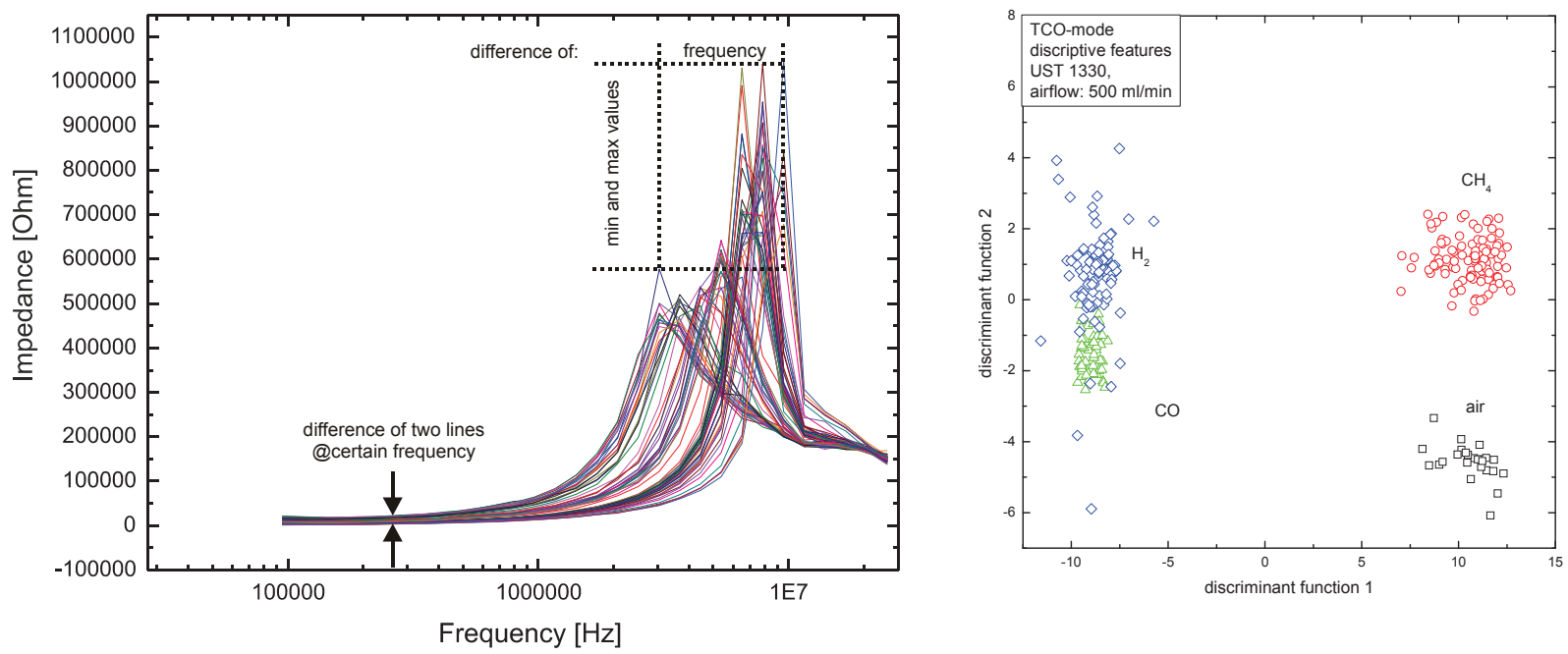

Fig. 9: Left: descriptive features from impedance spectra, right: LDA projection for the 3 test gases ( 2 concentrations each) and air based on descriptive features obtained from EIS in TCO mode.

Fig. 10 gives an overview over the new hardware concept for EIS based on the FFT approach. An FPGA (field programmable gate array) is used to generate the MLS sequence for sensor stimulation. A signal refining circuit is used to improve the spectral shape of the MLS signal and to allow amplification to match the system to specifications of different sc gas sensor, i.e. a maximum voltage across the measurement electrodes. We use bipolar signals to avoid DC currents across the sensor which might induce drift effects due to electromigration. A fast analog to digital converter (ADC) records both the stimulation signal as well as the sensor response in the time domain and stores the signals. The FPGA can then be used to calculate the spectra and the complex sensor impedance.

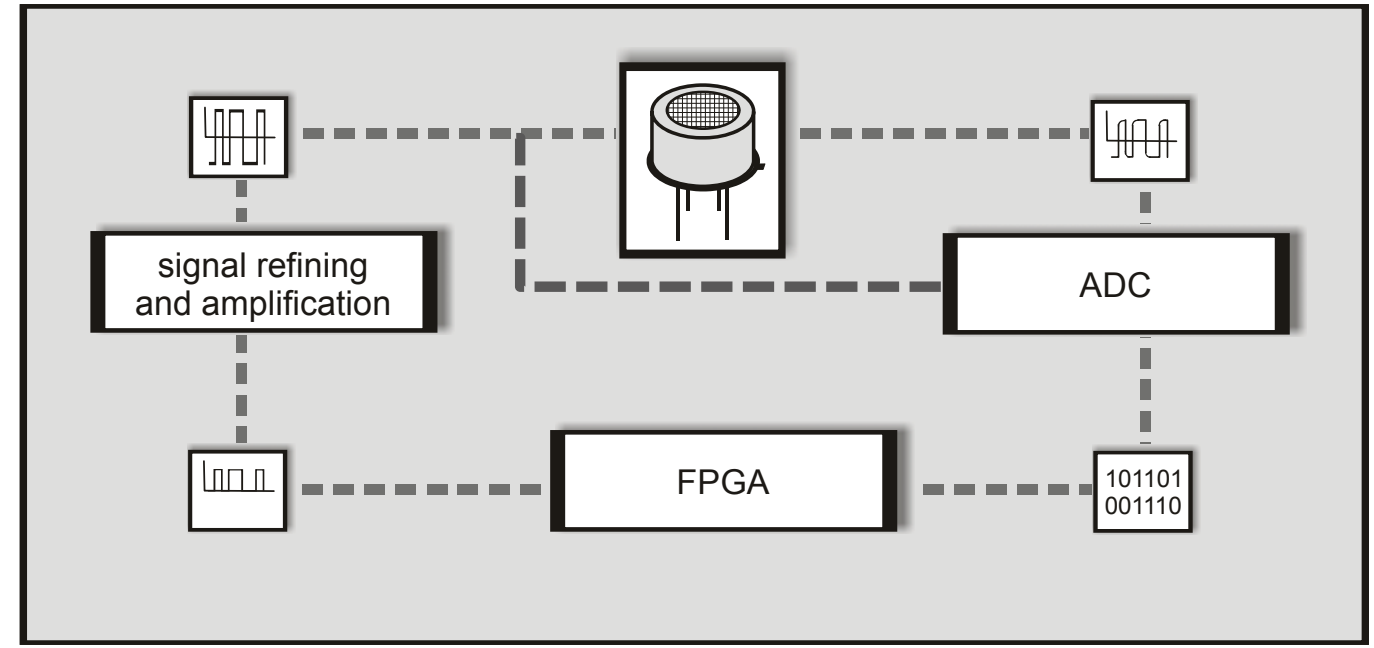

Fig.10: Overall hardware concept for the FPGA-based EIS hardware [17]

Fig. 11 shows the time domain and FFT spectra of the theoretical, simulated and real MLS signals and their FFT spectra, respectively. The ideal signal was generated by MATLAB, the simulated signal is produced by simulating the signal-refining amplifier circuit with an equivalent $L C R$ circuit in place of the gas sensor [5]. The third signal is the measured FPGA output. The simulated and measured signals show a good correlation with the ideal signal. The signal is based on a clock frequency of $200 \mathrm{MHz}$, allowing a minimum pulse length of $5 \mathrm{~ns}$, and a sequence with the length of 4095 . Figure 4 shows the corresponding energy spectrum in the frequency domain. It is not possible to use the whole frequency range because the spectral density goes down and reaches zero at $200 \mathrm{MHz}$. A useable maximum frequency is $100 \mathrm{MHz}$, at which the spectral density has decreased by $-3 \mathrm{~dB}$. 

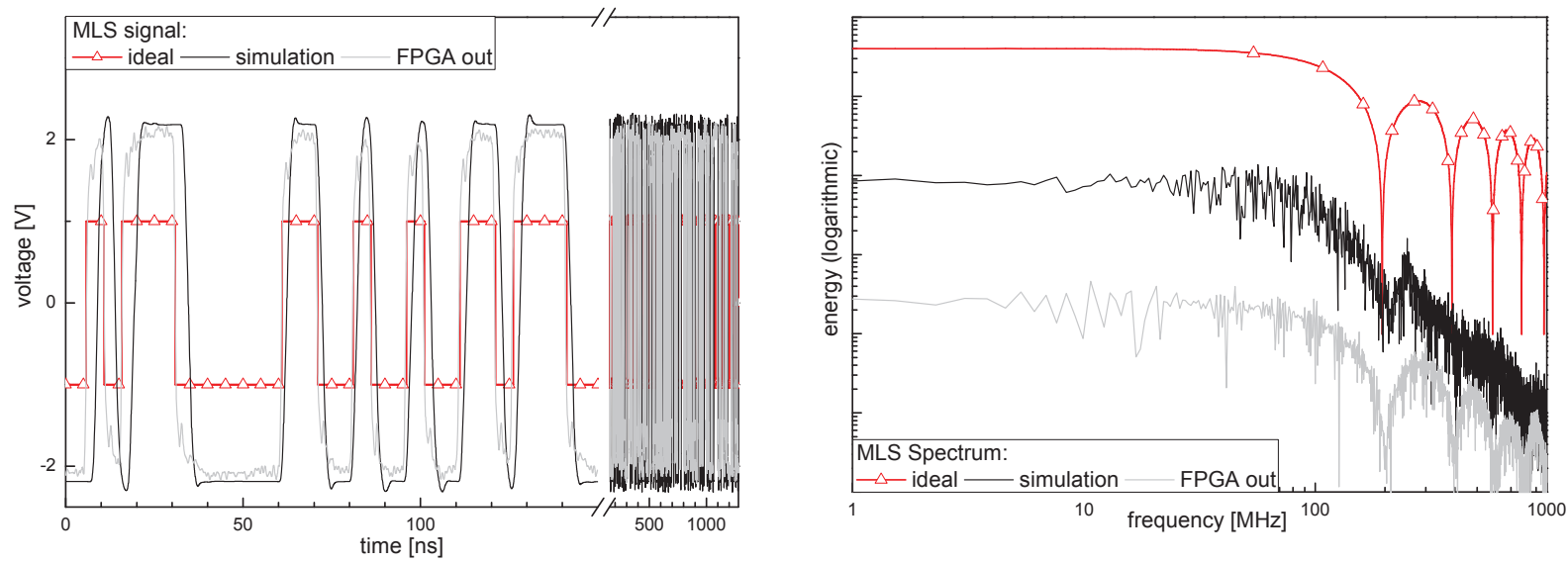

Fig. 11: left: MLS signals - ideal signal, signal simulation and FPGA output, right: Energy spectra of the MLS signals. The spectra are offset in the y-direction for better clarity.

\section{Conclusions and outlook}

In our FFT based approach the sensor impedance is calculated via sensor stimulation with MLS signals and obtaining the sensor response in the time domain. This approach has proven successful with standard lab equipment (signal generator for stimulation and oscilloscope for data acquisition). Based on this approach, we present a new hardware concept which allows the combination of TCO and EIS. The sensor stimulation and data acquisition are realized using an FPGA. First tests and simulations have shown promising results. In combination with the new hardware approach, significant primary and secondary features were extracted which allowing a good discrimination of different typical gases. In the next step, we plan to realize this hardware concept on a single PCB for comprehensive tests of the combined TCO/EIS mode not only in the lab but also in field test systems.

\section{References}

[1] U. Weimar, W. Göpel, "A.c. measurements on tin oxide sensors to improve selectivities and sensitivities", Sens. \& Act. B, 26-27, pp. 13-18, 1995.

[2] A.P. Lee, B.J. Reedy, "Temperature modulation in semiconductor gas sensing," Sens. \& Act. B, 60, 1999.

[3] P. Reimann, A. Dausend, A. Schütze; "A self-monitoring and self-diagnosis strategy for semiconductor gas sensor systems" Proc. IEEE Sensors Conference 2008, Lecce, Italy, Oct. 27 - 29, 2008.

[4] T. Gillen, P. Reimann. A. Schütze, "Feature extraction based on impedance spectroscopy for semiconductor gas sensors", International Workshop on Impedance Spectroscopy 2010, Chemnitz, Oct. 22-23, 2010.

[5] Conrad, T.; Reimann, P; Schütze, A.: "A hierarchical strategy for under-ground early fire detection based on a Tcycled semiconductor gas sensor", IEEE Sensors Conference, Atlanta, USA, Oct. 29-31, 2007.

[6] Gramm, A.; Schütze, A.: "High Performance Solvent Vapor Identification with a Two Sensor Array using Temperature Cycling and Pattern Classification", Sens. \& Act. B, 95, pp. 58-65, 2003.

[7] G. Sberveglieri, "Recent developments in semiconducting thin-film gas sensors", Sens. \& Act. B, 23, 1995.

[8] W. Göpel. K.D. Schierbaum, "SnO2 sensors: Current status and future prospects", Sens. \& Act. B, 26-27, 1995.

[9] T. Conrad, T. Fricke, P. Reimann, A. Schütze, "A versatile platform for the efficient development of gas detection systems based on automatic device adaptation", Proc. Eurosensors XX, Göteborg, Sweden, Sep. 1720, 2006

[10] P. Reimann, S. Horras, A. Schütze, "Field-test system for underground fire detection based on semiconductor gas sensors", Proc. IEEE Sensors Conf. 2009, Christchurch, NZ, October 25-28, 2009.

[11] T. Conrad, F. Trümper, H. Hettrich, A. Schütze: "Improving the Performance of Gas Sensor Systems by Impedance Spectroscopy: Application in Under-Ground Early Fire Detection”, Proc. SENSOR Conference 2007, Volume I, AMA Service GmbH, ISBN 978-3-9810993-1-7, pp. 169-174.

[12] P. Reimann, A. Dausend, A. Schütze; „Optimale Signalanregung zur Low-Cost Impedanzmessung von Halbleitergassensoren“ in: Sensoren und Messsysteme 2010, VDE Verlag, Berlin, Offenbach; ISBN 978-3-80073260-9.

[13] J. Schoukens, "System Identification - A Frequency Domain Approach", IEEE Press, 2001.

[14] UST Umweltsensortechnik GmbH, Geraberg, Germany; http://www.umweltsensortechnik.de

[15] Barsoukov, E.; Macdonald, J.R.: "Impedance Spectroscopy - Theory, Experiment and Applications", Wiley, 2005.

[16] R. Gutierrez-Osuna, "Pattern Analysis for Machine Olfaction: a Review," IEEE Sensors Journal, vol. 2, no. 3, pp. 189-202, 2002.

[17] M. Schüler, S. Darsch, P. Reimann; A. Schütze: "Low-cost impedance spectroscopy for semiconductor gas sensors - a hardware concept", International Workshop on Impedance Spectroscopy 2010, Chemnitz, Oct. 2223, 2010. 